\title{
AMENDMENTS
}

\section{Author Correction: Cas9 activates the p53 pathway and selects for p53-inactivating mutations}

Oana M. Enache, Veronica Rendo D, Mai Abdusamad, Daniel Lam, Desiree Davison, Sangita Pal, Naomi Currimjee, Julian Hess, Sasha Pantel, Anwesha Nag, Aaron R. Thorner, John G. Doench (D), Francisca Vazquez, Rameen Beroukhim (D), Todd R. Golub (D) and Uri Ben-David (D)

Correction to: Nature Genetics https://doi.org/10.1038/s41588-020-0623-4, published online 18 May 2020.

In the version of this article initially published, in Fig. 2a, the image showing the parental (WT) SNU466 cells was mistakenly a duplicate of the adjacent image of Cas9-expressing SNU466 cells. The image has been corrected, and the raw microscopy data have been made available at https://figshare.com/projects/Cas9_activates_the_p53_pathway_and_selects_for_p53-inactivating_mutations/81080. The 'Data availability' statement has been updated to include this URL. The SNU466 imaging data have consequently been reanalyzed, and the bar plots of $\gamma \mathrm{H} 2 \mathrm{AX}$-positive cells in Fig. $2 \mathrm{~b}$ have been replotted. The revised analysis yielded highly similar results to the original analysis: Cas9-expressing SNU466 cells exhibited a significantly higher fraction of $\gamma \mathrm{H} 2 \mathrm{AX}$-positive cells than their parental cells; the $P$ value for SNU466 has consequently been revised from 0.0041 to 0.009 . In all analyses, the parental and Cas9-expressing cells from each experiment were analyzed together, with the same fluorescence exposure and color intensity thresholds to enable a fair comparison. The original and corrected Fig. 2 panels are shown below. This error did not affect any other analyses or conclusions.

In Fig. 1d, the bands for EFM192A and MDAMB453 came from two different gels (which were run together): one for the housekeeping protein and one for the other proteins; only one gel was originally shown in the Source Data for Fig. 1. Figure 1d has been revised to show all the bands from a single gel, and the Source Data file for Fig. 1 has been updated accordingly. The original and corrected Fig. 1d is shown below. This error did not affect the protein-level quantification in Fig. 1e or any other analyses or conclusions.

In Extended Data Fig. 1d,e and Extended Data Fig. 3a, the code line used to generate the bar plots contained an error resulting in the fraction of lines in each category being divided by the number of WT cell lines instead of the total number of cell lines. These bar plots have been replotted. This error did not affect the statistical analyses or any other analyses or conclusions.

In the legend of Fig. 5d, 'Wilcoxon rank sum test' has been corrected to ' $t$-test'. In the Discussion, "We therefore compared Cas9 activity between 226 TP53-WT and 493 TP53-mutant lines" has been corrected to "We therefore compared Cas9 activity between 216 TP53-WT and 482 TP53-mutant lines," to match the numbers correctly described in Fig. 5a. The $P$ value in the legend of Extended Data Fig. 3a has been changed from 0.33 to 0.07 to match the value correctly described in the main text. The title of Extended Data Fig. 3 has been updated to indicate that it is related to Fig. 2 rather than Fig. 1; the title for Extended Data Fig. 4 has been updated to indicate that it is related to Fig. 3 rather than Fig. 2.

The errors have been corrected in the HTML and PDF versions of the article.

Original

d

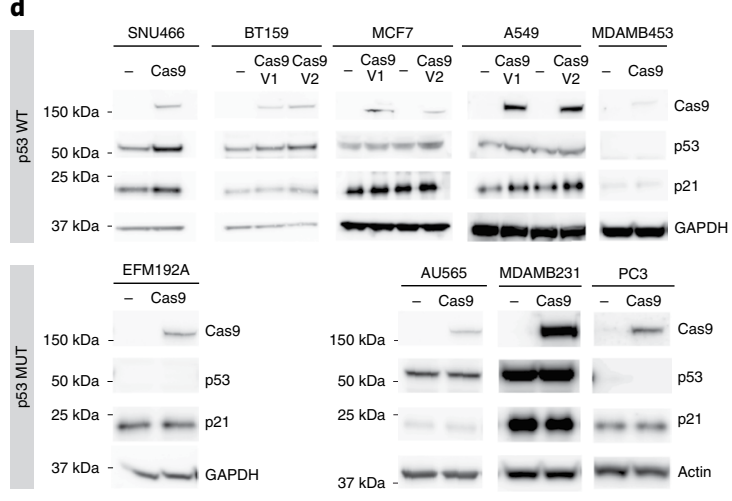

d

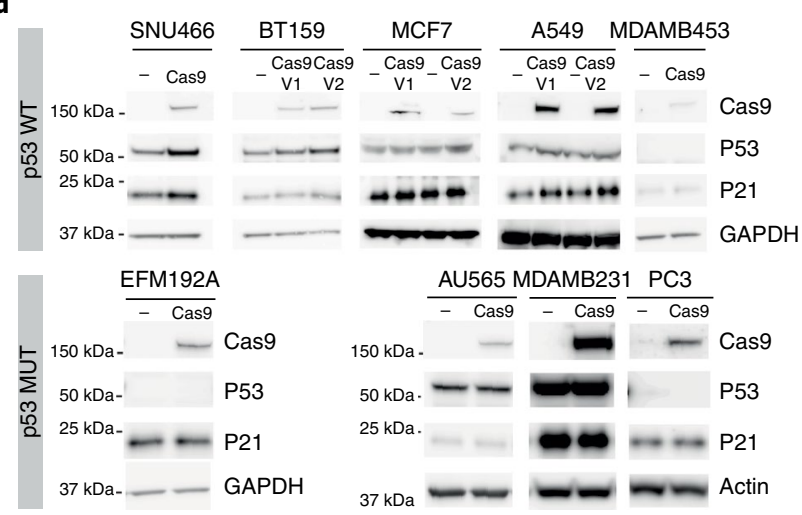

Fig. 1d | Original and corrected. 
Original
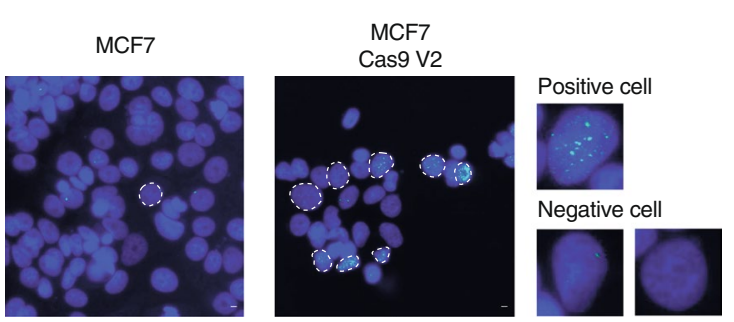

SNU466
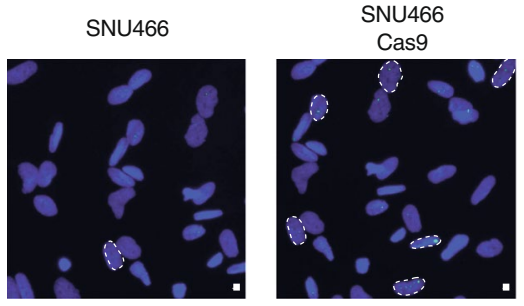

Hoechst $\gamma-\mathrm{H} 2 \mathrm{AX}$

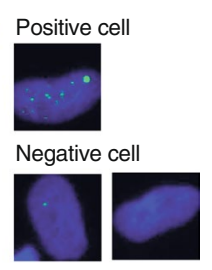

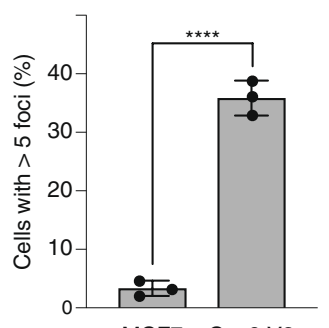

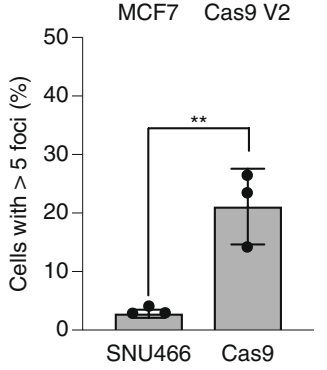

Corrected

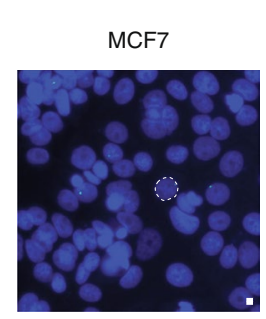

SNU466

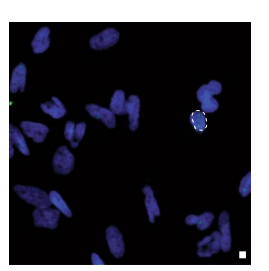

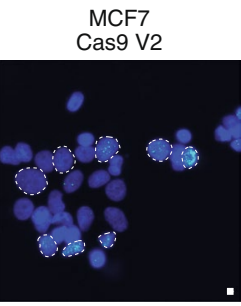

SNU466
Cas 9

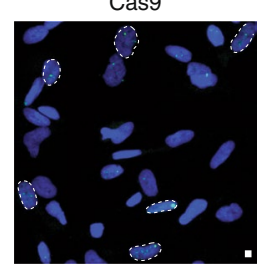

Hoechst $\gamma-\mathrm{H} 2 \mathrm{AX}$ b
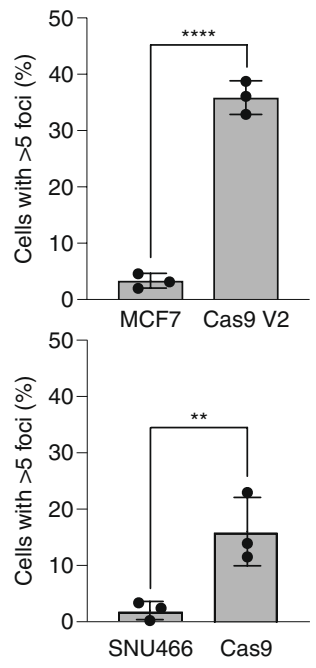

Fig. 2 | Original and corrected.

Published online: 25 June 2020

https://doi.org/10.1038/s41588-020-0663-9

(c) The Author(s), under exclusive licence to Springer Nature America, Inc. 2020 